\title{
Reply to comment on Wang et al.: operative versus nonoperative treatment for acute Achilles tendon rupture
}

\author{
Nan Jiang $\cdot$ Fu Dong $\cdot$ An-fu Chen $\cdot$ Bo-wei Wang $\cdot$ Bin Yu
}

Received: 10 June 2012 / Accepted: 10 June 2012 /Published online: 11 July 2012

(C) Springer-Verlag 2012

We would like to thank the readers of International Orthopaedics for their questions on our meta-analysis [1]. In response to these queries, we would like to make the following comments:

1. The readers advised that we should focus specifically on the search strategy. Thanks for their kind suggestion. We agreed with them that a comprehensive search was quite necessary in a meta-analysis. Although we did not list detailed index terms in the article, this did not mean that we neglected the role of search strategy. Two authors (NJ and $\mathrm{AFC}$ ) independently retrieved relevant studies by means of electronic search. It was probably one of the shortcomings that we did not list the index terms in detail.

2. The outcomes were just based on ten RCTs, of which most recruited patients were middle-aged. Whether the results are suitable for all kinds of patients needs further study. Therefore, a cautious approach should be taken towards the conclusions.

3. The readers suggested that the meta-analysis should be conducted through different surgical approaches. We agree that this might achieve a more accurate conclusion. Similarly, up to now, there are still several conservative methods of treating AATR. Therefore, future RCTs with consistent surgical approaches and conservative management are needed to evaluate detailed efficacies of operative and nonoperative AATR management.

4. Although the detailed scores were not listed for each study, we conducted a rigorous and comprehensive assessment of eight items for each RCT. The methodological assessment is a subjective activity and only provides a reference. With regard to the assessment method, one of the authors (FD) evaluated each RCT and the other (AFC) verified for accuracy. Discrepancies were resolved by consensus after discussion. We considered this would reduce subjective bias.

5. Results of our meta-analysis showed that operative AATR management might have a lower risk of rerupture than nonoperative treatment. We agree that medical costs and other risks might affect the clinical efficacy. However, there was still little effective available data and outcomes could not be pooled for meta-analysis regarding these measures based on current RCTs. Therefore, we hope there will be more RCTs concerning these measures in the future.

6. We agree that limited publication language may cause a bias. Therefore, on the one hand, as Sambunjak et al. [2] suggested that if at all possible, the study search should include non-English databases such as LILACs and SciELO for studies in Spanish and Portuguese, and the Chinese biomedical database Cnki.net. On the other hand, more high-quality RCTs are needed to reach more accurate conclusions.

\section{References}

1. Jiang N, Wang B, Chen A, Dong F, Yu B (2012) Operative versus nonoperative treatment for acute Achilles tendon rupture: a metaanalysis based on current evidence. Int Orthop 36:765-773

2. Sambunjak D, Franic M (2012) Steps in the undertaking of a systematic review in orthopaedic surgery. Int Orthop 36:477-484

N. Jiang $\cdot$ F. Dong $\cdot$ A.-f. Chen $\cdot$ B.-w. Wang $\cdot$ B. Yu $(\bowtie)$

Department of Orthopaedics and Traumatology, Nanfang Hospital,

Southern Medical University,

Guangzhou 510515, People's Republic of China

e-mail: nanfanghot@126.com 